\title{
The Latin American Giant Observatory (LAGO) capabilities for detecting Gamma Ray Bursts
}

\section{Christian Sarmiento-Cano, ${ }^{a, *}$ H. Asorey, ${ }^{a}$ J.R. Sacahui, ${ }^{b}$ L. Otiniano ${ }^{c}$ and I. Sidelnik ${ }^{d}$ on behalf of the LAGO Collaboration}

(a complete list of authors can be found at the end of the proceedings)

${ }^{a}$ Instituto de Tecnologías en Detección y Astropartículas (ITeDA, CNEA/CONICET/UNSAM)

Centro Atómico Constituyentes, Av. General Paz 1499, 1450 Villa Maipú, Buenos Aires, Argentina

${ }^{b}$ Instituto de Investigación en Ciencias Físicas y Matemáticas, USAC, Ciudad Universitaria zona 12, Guatemala, Guatemala

${ }^{c}$ Comisión Nacional de Investigación y Desarrollo Aeroespacial, CONIDA, Lima, Perú

${ }^{d}$ Departamento de Física de Neutrones, Centro Atómico Bariloche (CNEA/CONICET) Av. Bustillo 9500, 8400 San Carlos de Bariloche, Argentina

E-mail: christian.sarmiento@iteda.cnea.gov.ar

The Latin American Giant Observatory (LAGO) consists of a network of small water Cherenkov detectors (WCD) located at different sites in Latin America. It is a large aperture observatory sensitive to high energy gamma rays and due to its high duty cycle, LAGO constitutes a facility to detect transient events from the ground. Gamma Ray Bursts (GRBs) are of the brightest transients detected, with typical energies in their prompt phase ranging from $\mathrm{keV}$ to $\mathrm{MeV}$, but theoretical models predict emissions at higher energies in the early times of the afterglow emission, and recently GRB190114C was the first GRB detected at TeV energies by the MAGIC experiment. In this work, we present the results of the expected sensitivity of LAGO for possible events like GRB190114C. We performed simulations in four of the high altitude LAGO sites projected to assess the sensitivity of the Observatory for this kind of events, using the ARTI toolkit developed by LAGO. We simulate photon showers with different spectral slopes and energies from $200 \mathrm{GeV}$ to $1 \mathrm{TeV}$ using the parameters presented by MAGIC for the recorded event. We also present maps of field of view of the studied sites with the observed GRBs by Fermi-GBM from 2019 to 2021.

$37^{\text {th }}$ International Cosmic Ray Conference (ICRC 2021)

July 12 th - 23rd, 2021

Online - Berlin, Germany

\footnotetext{
*Presenter
} 


\section{Introduction}

The LAGO Collaboration is a non-centralized and distributed network of more than 100 scientists from institutions from Argentina, Bolivia, Brazil, Chile, Colombia, Ecuador, Guatemala, México and Perú and Spain. The collaboration operates a detection network consisting of water Cherenkov detectors (WCD) deployed at ground level, spanning over different sites located at significantly different latitudes (currently planned from México to the Antarctic region) and different altitudes (from sea level to more than $5000 \mathrm{~m}$ a.s.l.), covering a wide range of geomagnetic rigidity cut off limits and atmospheric absorption / reaction levels.

One of the objectives of LAGO is the observation of the extreme universe through the study of air showers generated by high energy gamma rays. We do this by looking for excess in the secondary particle flux measured by WCDs located at the highest altitude sites, examples of these sites are Chacaltaya (Bolivia) and Sierra Negra (México). Due to the wide Field of View (FOV) of LAGO, it constitutes a good facility for monitoring transient events like Gamma Ray-Bursts (GRBs), that are among the brightest events detected. Common GRBs present an initial short phase, the called prompt emission phase, with typical energies between $\mathrm{keV}-\mathrm{MeV}$, followed by a longer afterglow emission. Recently the MAGIC Collaboration reported for GRB 190114C the first unambiguous detection from the ground of a GRB at energies between $200 \mathrm{GeV}$ to $1 \mathrm{TeV}$ [1]. Its flux was detected at more than 50 standard deviations from background in its first 20 minutes. Also, around ten hours after the prompt emission, the H.E.S.S. collaboration [2] reports the emission from GRB 180720B in the $100-440 \mathrm{GeV}$ energy band. These two bursts confirms emissions from GRBs with energies up to several $\mathrm{GeV}$.

As during the occurrence of a GRB a sudden increase in the flux of primary photons reaching the atmosphere is observed, LAGO has shown that the single particle technique (SPT) is feasible for the observation of increases in expected secondary cosmic ray counts in single and small arrays of WCDs [3, 4]. Previous simulations of photon-initiated air shower in the energy range of $1 \mathrm{GeV}$ to $1 \mathrm{TeV}$ showed a significant amount of electromagnetic particles arriving with energies averaging $10 \mathrm{MeV}$ for the Chacaltaya and Sierra Negra sites. By looking for excesses in $5 \mathrm{~ms}$ window, this study find no coincidences with observed GRBs[5].

Later, in [4], the scalers rates (an implementation of the SPT) of the LAGO WCDs installed in Mount Chacaltaya were analyzed. This analysis was performed searching for transient increases in the counting rates, for short transient events ( $\Delta T<1$ minute). After processing the data, a potential candidate was found on Wednesday Dec 07-15: 47: 02.378 UTC 2011. However, no coincidences were found with events recorded in the SWIFT and Fermi satellites and also in the base of data from the Gamma Ray Coordinate Network (GCN).

Currently, LAGO has completed a framework of codes called ARTI[6], a simulation framework that goes from the primary CR to the estimation of the response of the detectors, including the estimation of the background radiation for each LAGO site. In addition, an update to the acquisition electronics [7, 8], would allow a higher resolution in the energy spectra measured by a WCD, is under way. In these contexts and in the recent detection of MAGIC, we updated the first stage of the LAGO simulation chain for the study of GRBs. 


\section{LAGO High Altitude Sites}

High altitude LAGO sites ( $h>4000 \mathrm{~m}$ ) are designed and operated mainly for the search of high energy components of GRB Such sites are chosen to diminish the atmospheric absorption. To increase electromagnetic-muon separation at a single pulse level in those sites, a method based on the total charge and pulse rise-time analysis is also implemented. The increase in separation performance can be used to improve the search for possible GRB candidates, as gamma-initiated cascades show lower muon fractions at the detector level when compared with hadronic primaries. Due to electromagnetic particles are the most affected component due to atmospheric absorption, for these studies high altitudes sites are preferred.

Since LAGO spans from México to Antarctica, for the evaluation of the high energy component of GRBs we focused this work in five of the highest sites of the network. In the table 1 we show the characteristics and locations of these high-altitude sites. In [6] a list of all the LAGO sites as long as their geographical distribution and the correspondent rigidity cut off can be seen.

\begin{tabular}{|c|c|c|c|c|}
\hline Country & Site & $\begin{array}{c}\text { Altitude } \\
{[\mathrm{m} \text { a.s.l. }]}\end{array}$ & $\begin{array}{c}\text { Latitude } \\
{[\mathrm{deg}]}\end{array}$ & $\begin{array}{c}\text { Longitude } \\
{[\mathrm{deg}]}\end{array}$ \\
\hline \hline Argentina & SAC & 4500 & $24.23 \mathrm{~S}$ & $66.32 \mathrm{~W}$ \\
\hline Chile & Atacama & 5100 & $23 \mathrm{~S}$ & $67.76 \mathrm{~W}$ \\
\hline Ecuador & Chimborazo & 5000 & $1.47 \mathrm{~S}$ & $78.82 \mathrm{~W}$ \\
\hline Perú & Imata & 4600 & $15.84 \mathrm{~S}$ & $71.10 \mathrm{~W}$ \\
\hline
\end{tabular}

Table 1: Location of the LAGO sites used in this work, with the highest altitudes of the Observatory. It can be seen each site country, altitude and coordinates. We evaluate the prospect of this four projected LAGO sites and the feasibility to be used to study the high energy component of GRBs.

The selected sites corresponds to the highest sites selected for the operation of LAGO WCDs. Imata are about to be deployed and San Antonio de los Cobres (SAC), Atacama and Chimborazo are in good perspective to accommodate a single WCD or an array.

\section{LAGO Field of View}

The Gamma-Ray Burst Monitor (GBM) is the instrument on board the Fermi Observatory dedicated to the study of GRBs [9]. It is sensitive to photons in the energy range of $8 \mathrm{keV}$ to 40 $\mathrm{MeV}$. GRBs are detected from all directions in the sky and GBM reports a rate of detection of $\sim 240$ GRBs per year [10]. In Fig. 1 we present the bursts observed by GBM in the years 2019, 2020 up to May 2021. We present in red the GRBs that were in the field of view (FOV) of the above described LAGO high altitude sites (above $4500 \mathrm{~m}$ a.s.l.) presented in table 1. The dotted lines represents the path of the zenith of each detector in the sky, and the FOV band is projected in the colored bands for each site by considering a cone $15^{\circ}$ cone respect to the local zenith. Ten burst were within the FOV of LAGO during the year 2019, 13 burst during 2021 and 4 bursts in the first 4 months of 2021. When all the detectors together are considered, a wide FOV is reached, with a good coverage of the galactic plane, making LAGO a great facility for this king of high energy GRBs. 

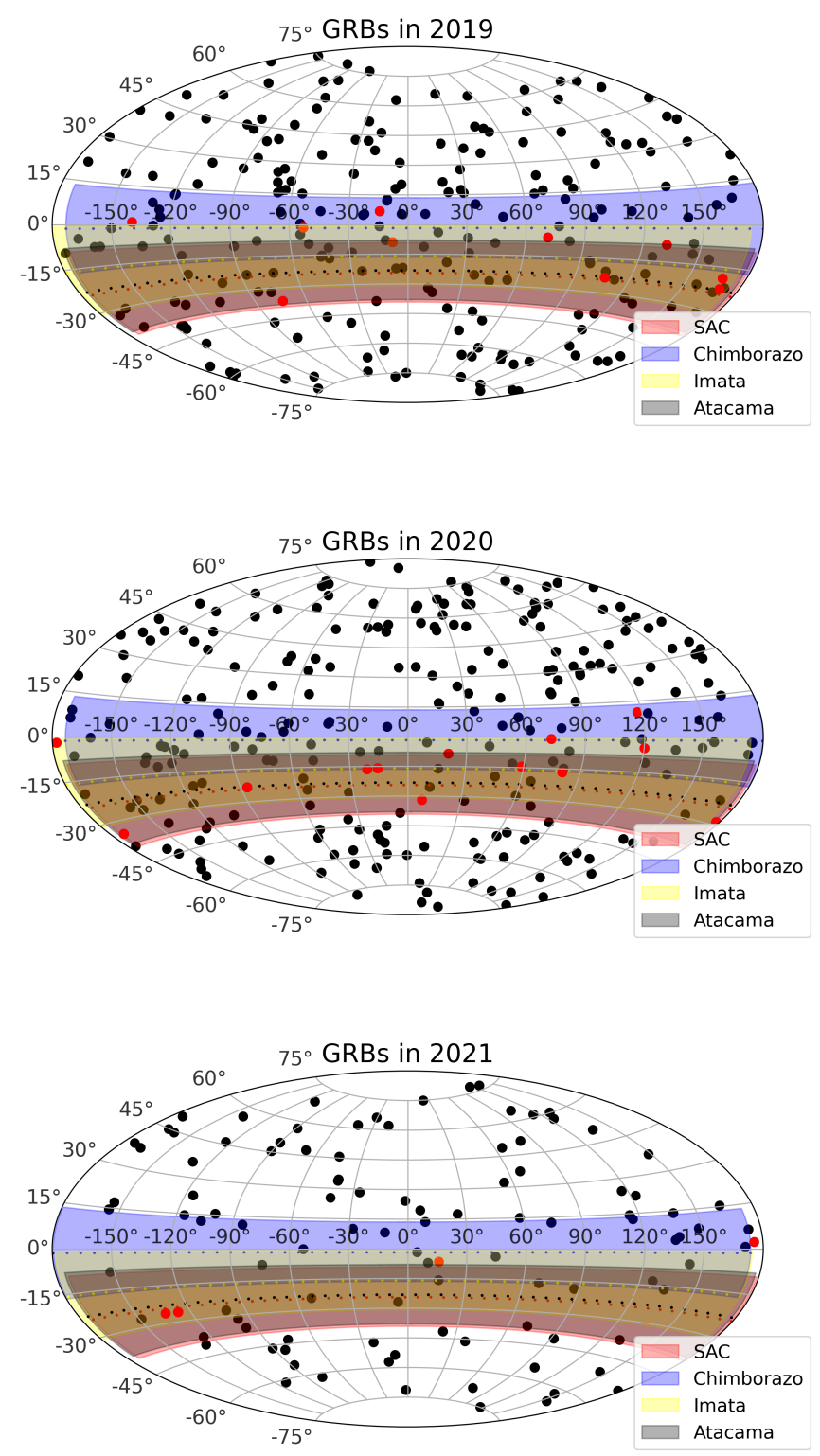

Figure 1: GRBs detected by Fermi-GBM in the years 2019, 2020 and up to May 2021. We included the field of view of the LAGO high altitude sites described in the Table 1. We highlighted in red the GRBs that were in the FOV of these LAGO sites within a cone of $15^{\circ}$ centred at the local zenith. Coloured doted lines represents the pass of the zenith of each site in the sky and the coloured bands represents $15^{\circ}$ aperture cone.

\section{Methodology}

All the simulations were performed using ARTI, as it is specifically designed to take into account the locations of different sites, including the variety of geomagnetic rigidity cutoffs, the atmospheric absorption (site height), the type of atmosphere and other characteristics [6, 11, 12]. The energy range and spectral index were taken from the events detected by the MAGIC observatory 
and reported in [1]. Therefore, the energy range used was $0.2 \mathrm{TeV} \leq \mathrm{E}_{p} \leq 1 \mathrm{TeV}$ As for the $\alpha$ spectral index, three different values were used, $\alpha_{1}=-2.2, \alpha_{2}=-2.7$ y $\alpha_{3}=-3.37$.

Considering that the GRB emission is composed of gamma photons with energy range $0.2 \mathrm{TeV}-1 \mathrm{TeV}$. To build our model we defined three energy intervals, $0.2 \mathrm{TeV} \leq \mathrm{E}_{p} \leq 0.4 \mathrm{TeV}$, $0.4 \mathrm{TeV} \leq \mathrm{E}_{p} \leq 0.7 \mathrm{TeV}$ y $0.7 \mathrm{TeV} \leq \mathrm{E}_{p} \leq 1 \mathrm{TeV}$, as shown in the vertical lines of Fig. 2 (a).

Then, for each interval and $\alpha_{i}$, we produce 50,000 $\gamma$-initiated EAS and computed the flux of secondaries arriving at the ground, and the avreage was weighted by the contribution of each component to the total flux. For example, the weights for the $\alpha=-2.2$ case are respectively $w_{1}=0.66, w_{2}=0.25$, and $w_{3}=0.09$ at each energy band.

Fig. 2 (b) shows the particle flux against secondaries energy for the three considered values of $\alpha$. As can be seen, the three have very similar behavior, this is due to the fact that the component $0.2 \mathrm{TeV} \leq \mathrm{E}_{p} \leq 0.4 \mathrm{TeV}$ has a strong influence and the third one, $0.7 \mathrm{TeV} \leq \mathrm{E}_{p} \leq 1 \mathrm{TeV}$, does not have enough weight to modify the total secondary flux.

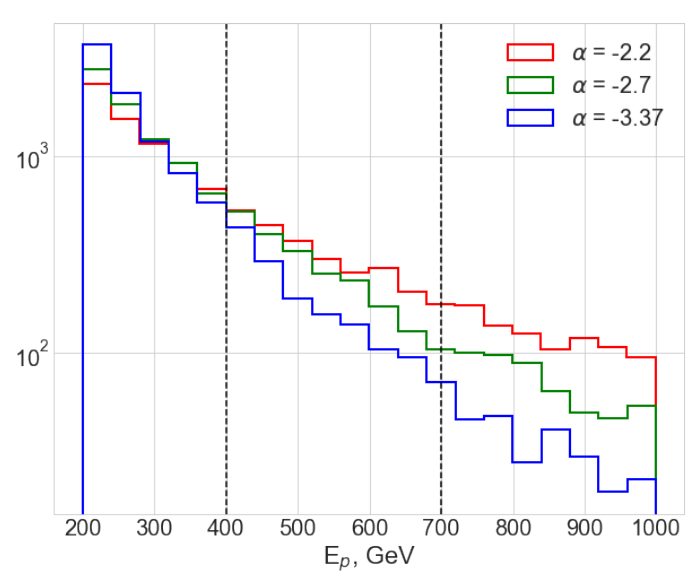

(a)

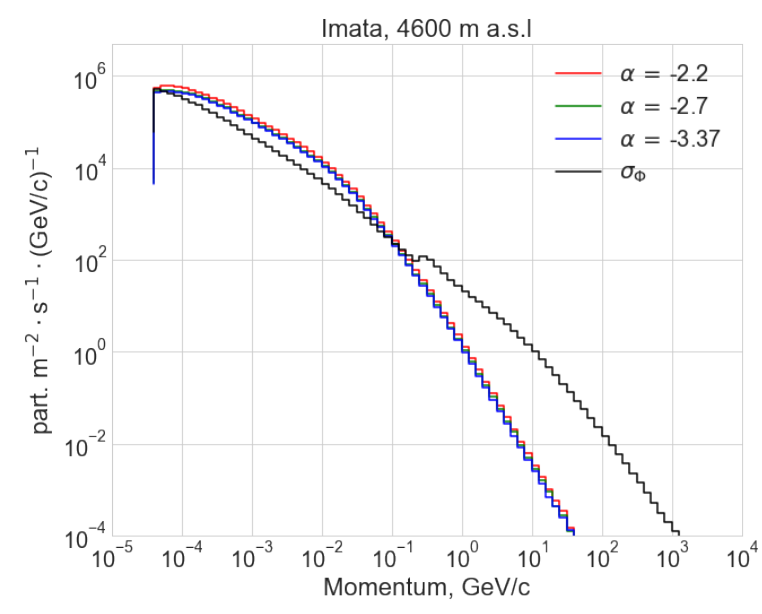

(b)

Figure 2: (a) Energy distribution for the three alpha used in this work. As can be seen, the components change with respect to the $\alpha$ value, as expected. (b) Flux at detector level generated by the GRB composition using the three energy intervals in each of the $\alpha$ index used.

\section{Results and Conclusive Remarks}

The methodology described in section 4 was applied to the following LAGO sites: Imata (Peru), Atacama (Chile), San Antonio de los Cobres (Argentina), and Chimborazo (Ecuador). In this case, the analysis was extended to primary gamma photons with zenith angles of $0^{\circ}$ and $15^{\circ}$. Fig. 3 shows the comparison between the background flux and the secondary fluxes generated by the GRBs modeled in each site studied. As can be seen in all cases, the flux exceeds the background, and the difference between them becomes greater as the height above sea level increases. No significant differences were noticed between events with zenith angle of $0^{\circ}$ and $15^{\circ}$, as the secondary flux of particles generated by primary photons is relatively constant with the zenith angle up to $30^{\circ}$. However, for $\theta \gtrsim 30^{\circ}$, even at high altitude sites the flux of secondary particles decrease steeply. 


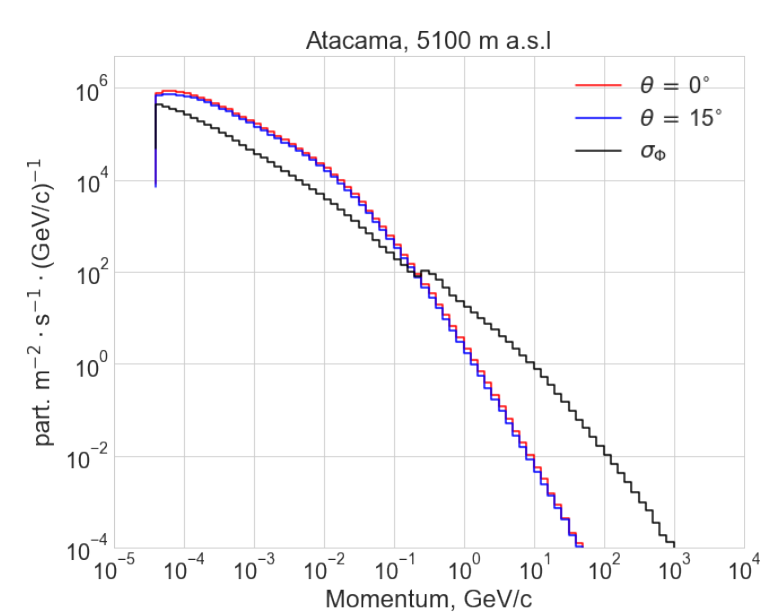

(a)

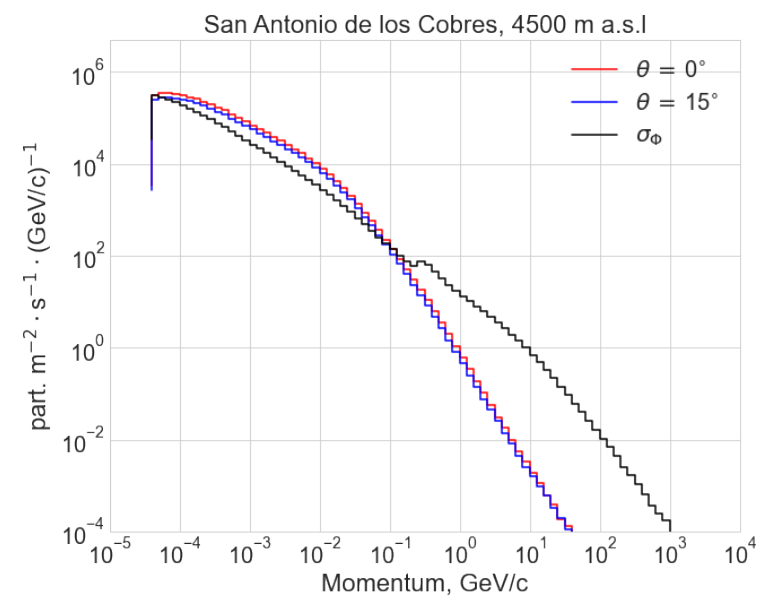

$(c)$

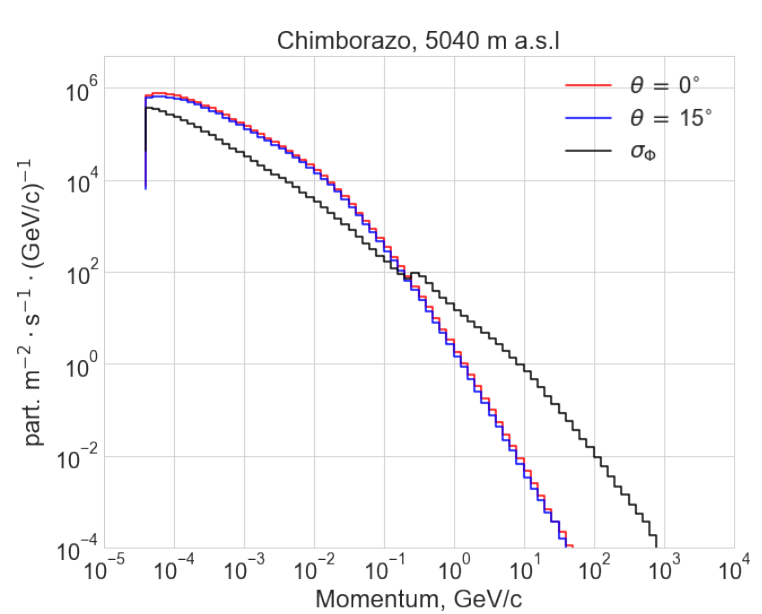

(b)

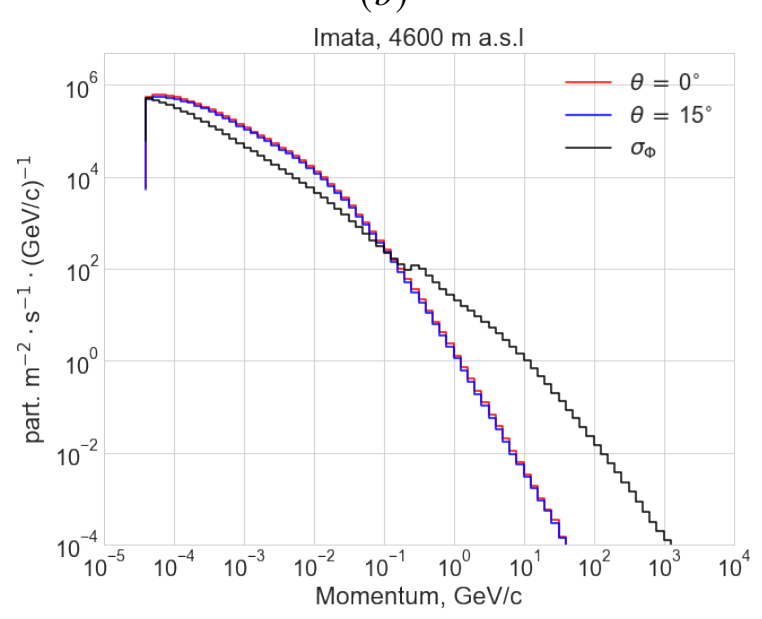

(d)

Figure 3: Comparison between the background expected fluctuation and the secondary flux generated by GRB injected at zenith angles of 0 and 15 degrees at four high altitude (> $4000 \mathrm{~m}$ a.s.l.) LAGO sites: Atacama, Chimborazo, San Antonio de los Cobres, and Imata. As can be observed, in all cases and specially for Atacama, in the low energy sector the increase in the flux is significantly greater than the expected fluctuation of the background.

As explained in the section 1, the implementation of the SPT in our detectors implies that each one of the LAGO detectors is continuously measuring the background at an almost single particle basis, i.e., within the detector efficiency we are able to measure every charged particle with enough energy to produce Cherenkov radiation above our detection threshold at a $300 \mathrm{~ns}$ basis. Moreover, with the new LAGO electronics [8], or by improving our standard analysis by means of machine learning techniques (currently under development), we can reduce that time interval to the $\sim 100 \mathrm{~ns}$ scale. Additionally, massive detectors like our WCDs are able to measure the EAS photon component trough Compton and pair-creation processes inside the water volume as they produce charged leptons above the Cherenkov threshold [13]. All this setup allows us to have a very precise determination of the behaviour of the flux of secondary particles at each one of our detectors. Given 
the poissonian nature of the secondary flux $\Phi(t)$, non-poissonian deviations can be easily identified as excesses or deficits in the rate of particles, and so, we only need to assure that our detectors are capable to find an increase in the counting rate over the expected background fluctuation, namely, $\Delta \Phi(t)>\sigma_{\Phi} \stackrel{\text { def }}{=} \sqrt{\langle\Phi(t)\rangle}$, at a-priori defined levels of significance. This is what we show in the Figure 3, where we compare the expected fluctuation of the secondary flux at the detector with the secondaries originated by a typical weight-averaged EAS produced by a single GRB primary photon. For a typical photon originated by a GRB, at the $\sim 10 \mathrm{MeV} / \mathrm{c}$ secondary momentum scale where the flux is dominated by the EM component, large deviation from the background baseline of up to $\gtrsim 5 \sigma_{\Phi}$ is expected. ${ }^{1}$ These figures are compatible with the previously reported GRB candidate at Chacaltaya by looking for excesses on the low energy scalers rates [4]. Statistical errors of the synthetic background are extremely reduced given the large integration times of the calculated fluxes by using the new LAGO-ARTI cloud implementation [6]. As an example, for Imata we integrate $\sim 2.3 \times 10^{9}$ secondaries, corresponding to a total integration time of $t=345,600 \mathrm{~s}$ (4 days) per squared meter for the whole hemisphere above a volumetric detector [15].

Furthermore, after a careful inspection of the lateral distributions at ground of the energy density of GRBs-originated EAS, it is possible to conclude that given the uniformity of the photon flux impinging the Earth atmosphere, the distribution of secondaries at ground originated by a single primary photon not impinging in a much smaller detector than the footprint at ground, will be compensated by secondaries originated by neighbours primaries, just as it happens with the background primary flux.

While the expected flux of primary photons originated during the prompt phase of the GRBs is low, we show the LAGO capabilities for the detection of the highest energy component of long GRBs at the high altitude sites ( $h>4000 \mathrm{~m}$ a.s.l.) of our detection network.

In this work, we have revisited the detection capabilities of the LAGO network at high altitude sites ( $h>4000 \mathrm{~m}$ a.s.l.) of the high energy component of the GRBs, based on recent observations of GRB photons with energy up to $\lesssim 1 \mathrm{TeV}$, and new developments on the synthetic reproduction of EAS developments, including new high and low energy interaction models, realistic atmospheric profiles and improved detector responses.

At this moment, new detectors are being developed and will be installed in the 2022 summer campaign in some selected sites at Peru, Chile, Ecuador and Argentina.

If such a high energy long GRB occurs within our field of view in these new detection sites, large deviations from the secondary particle flux baseline are expected in our detectors, even at the $>5 \sigma_{\Phi}$ level.

\section{Acknowledgements}

CSC, IS and HA acknowledge the support of CONICET and CNEA. This work was partly carried out within the 'European Open Science Cloud - Expanding Capacities by building Capabilities' (EOSC-SYNERGY) project, founded by the European Commission' Horizon 2020 RI Programme under Grant Agreement $n^{\circ} 857647$. This work has been also partially carried out on the ITeDA

\footnotetext{
${ }^{1}$ For a calibrated WCD, the detection efficiency depends on the energy and type of the particles impinging the detector. We observed the major differences where the flux is dominated by the EM component. At this level, signals produced by clipping corners muons are at the level of a few percent. See [6] and [14] for further details.
} 
cluster, we thank A.P.J. Sedoski Croce for his continuous support.

The LAGO Collaboration is very thankful to all the participating institutions and to the Pierre Auger Collaboration for their continuous support.

\section{References}

[1] MAGIC Collaboration, V.A. Acciari, S. Ansoldi, L.A. Antonelli, A. Arbet Engels, D. Baack et al., Teraelectronvolt emission from the $\gamma$-ray burst GRB 190114C, Nature 575 (2019) 455 [2006.07249].

[2] H. Abdalla, R. Adam, F. Aharonian, F. Ait Benkhali, E.O. Angüner, M. Arakawa et al., A very-high-energy component deep in the $\gamma$-ray burst afterglow, Nature 575 (2019) 464 [1911.08961].

[3] D. Allard, C. Alvarez, H. Asorey, H. Barros, X. Bertou, M. Castillo et al., Water cherenkov detectors response to a gamma ray burst in the large aperture grb observatory, arXiv preprint arXiv:0906.0820 (2009) .

[4] H. Asorey, P. Miranda, A. Núñez-Castiñeyra, L. Núñez, J. Salinas, C. Sarmiento-Cano et al., Analysis of background cosmic ray rate in the 2010-2012 period from the lago-chacaltaya detectors, Proceedings of Science, The Hague (2015) .

[5] D. Allard, I. Allekotte, C. Alvarez, H. Asorey, H. Barros, X. Bertou et al., Use of water-cherenkov detectors to detect gamma ray bursts at the large aperture grb observatory (lago), Nuclear Instruments and Methods in Physics Research Section A: Accelerators, Spectrometers, Detectors and Associated Equipment 595 (2008) 70.

[6] A. Rubio-Montero, R. Pagán-Muñoz, R. Mayo-García, A. Pardo-Diaz, I. Sidelnik and H. Asorey, The EOSC-Synergy cloud services implementation for the Latin American Giant Observatory (LAGO), (This proceedings), 2021.

[7] L. Arnaldi, D. Cazar, M. Audelo and I. Sidelnik, The new data acquisition system of the lago collaboration based on the redpitaya board, in 2020 Argentine Conference on Electronics (CAE), pp. 87-92, IEEE, 2020.

[8] LAGO collaboration, Preliminary results of the design and development of the data acquisition and processing system for the lago collaboration, in 36th ICRC, (Madison, WI, U.S.A.), p. PoS(ICRC2019)175, 2019.

[9] C. Meegan, G. Lichti, P.N. Bhat, E. Bissaldi, M.S. Briggs, V. Connaughton et al., The Fermi Gamma-ray Burst Monitor, Astroparticle Journal 702 (2009) 791 [0908.0450].

[10] A. von Kienlin, C.A. Meegan, W.S. Paciesas, P.N. Bhat, E. Bissaldi, M.S. Briggs et al., The Fourth Fermi-GBM Gamma-Ray Burst Catalog: A Decade of Data, Astroparticle Journal 893 (2020) 46 [2002 . 11460].

[11] LAGO collaboration, Performance of the LAGO water Cherenkov detectors to cosmic ray flux, 2010.14591.

[12] LAGO collaboration, Modeling the LAGO's detectors response to secondary particles at ground level from the Antarctic to Mexico, in 36th ICRC, (Madison, WI, U.S.A), p. PoS(ICRC2019)412, 2019, https://pos.sissa.it/358/412/pdf.

[13] I. Sidelnik, H. Asorey et al., Simulation of 500 mev neutrons by using nacl doped water cherenkov detector, Advances in Space Research $\mathbf{6 5}$ (2020) 2216.

[14] L. Otiniano, H. Asorey, C. Sarmiento-Cano, I. Sidelnik, M. Suarez-Durán and the LAGO Collaboration, Influence of Simultaneous particles on the LAGO's Water Cherenkov Detectors, in 37th ICRC, no. 267, (Berlin, Germany), pp. 1-4, 2021.

[15] H. Asorey, L.A. Núñez and M. Suárez-Durán, Preliminary results from the latin american giant observatory space weather simulation chain, Space Weather 16 (2018) 461 [https://agupubs.onlinelibrary.wiley.com/doi/pdf/10.1002/2017Sw001774]. 


\section{Full Authors List: LAGO LAGO Collaboration}

V. Agosín ${ }^{20}$, A. Alberto ${ }^{3}$, C. Alvarez ${ }^{16}$, J. Araya ${ }^{20}$, R. Arceo ${ }^{16}$, O. Areso ${ }^{13}$, L. H. Arnaldi ${ }^{2}$, H. Asorey ${ }^{14,7}$, M. Audelo ${ }^{9}$, M.G. Ballina-Escobar ${ }^{19}$, D. C. Becerra-Villamizar ${ }^{18}$, X. Bertou ${ }^{2}$, K.S. Caballero-Mora ${ }^{16}$, R. Caiza ${ }^{8}$, R. Calderón-Ardila ${ }^{14}$, Calle, J. ${ }^{24}$, A. C. Fauth $^{27}$, E. Carrera Jarrin ${ }^{26}$, L. E. Castillo Delacroix ${ }^{11}$, C. Castromonte ${ }^{25}$, Cazar-Ramírez D. ${ }^{26}$, Diego Cogollo ${ }^{28}$, D. A. Coloma Borja $^{26}$, R. Conde ${ }^{1}$, J. Cotzomi ${ }^{1}$, D. Dallara ${ }^{11}$, S. Dasso ${ }^{13,5,6}$, R. Aguiar ${ }^{27}$, Albuquerque, A. ${ }^{28}$, J.H.A.P.Reis ${ }^{27}$, H. De León ${ }^{16}$, R. deLeón-Barrios ${ }^{23}$, D. Domínguez ${ }^{8}$, M. Echiburu ${ }^{21}$, M. González ${ }^{2}$, M. Gómez Berisso ${ }^{2}$, J. Grisales Casadiegos ${ }^{23}$, A. M. Gulisano ${ }^{13,12,6}$, Juan Carlos Helo ${ }^{17}$, Condori, C. A. H. ${ }^{24}$, J. E. Ise ${ }^{11}$, Nascimento, G. K. ${ }^{28}$, M. A. Leigui de Oliveira ${ }^{29}$, F. L. Miletto ${ }^{27}$, V. P. Luzio ${ }^{29}$, F. Machado ${ }^{25}$, Juan F. Mancilla-Caceres ${ }^{22}$, D. Manriquez ${ }^{20}$, A. Martínez-Méndez ${ }^{23}$, O. Martinez ${ }^{1}$, R. Mayo-García ${ }^{3}$, L.G. Mijangos ${ }^{22}$, Miranda, $\mathrm{P}^{24}$, M. G. Molina ${ }^{11}$, I.R. Morales ${ }^{19}$, O.G Morales-Olivares ${ }^{16}$, E. Moreno-Barbosa ${ }^{1}$, P. Muñoz ${ }^{17}$, Nina, $^{24}$, L.A. Núñez ${ }^{23}$, L. Otininano ${ }^{4}$, R. Pagán-Muñoz ${ }^{3}$, K. M. Parada-Jaime ${ }^{18}$, H. M. Parada-Villamizar ${ }^{18}$, R. Parra $^{10}$, J. Peña-Rodríguez ${ }^{23}$, M. Pereira $^{13}$, Y. A. Perez-Cuevas ${ }^{18}$, H. Perez ${ }^{19}$, J. Pisco-Guabave ${ }^{23}$, Raljevic, M. ${ }^{24}$, M. Ramelli ${ }^{13}$, C. Ramírez ${ }^{22}$, Rivera, ${ }^{24}$, L. T. Rubinstein ${ }^{13}$, A.J. Rubio-Montero $^{3}$, J.R. Sacahui ${ }^{19}$, H. Salazar ${ }^{1}$, N. Salomón ${ }^{11}$, J. Samanes ${ }^{4}$, N.A. Santos ${ }^{5}$, C. Sarmiento-Cano ${ }^{14}$, I. Sidelnik ${ }^{2}$, Mayra B. Silva $^{22}$, O. Soto ${ }^{17}$, M. Suárez-Durán ${ }^{18,31}$, Subieta Vasquez, M. ${ }^{24}$, Terrazas C. ${ }^{24}$, Ticona, R. ${ }^{24}$, T. Torres Peralta ${ }^{11}$, Pablo A. Ulloa ${ }^{17}$, Z.R. Urrutia ${ }^{22}$, N. Vásquez ${ }^{8}$, A. Vázquez-Ramírez ${ }^{23}$, A. Vega ${ }^{20}$, P. Vega ${ }^{17}$, J. Vega $^{4}$, A. Vesga-Ramirez ${ }^{14}$, D. Vitoreti ${ }^{30}$, R. Wiklich Sobrinho ${ }^{29}$,

${ }^{1}$ Benemérita Universidad Autónoma de Puebla". ${ }^{2}$ Centro Atómico Bariloche (CNEA/CONICET/IB)". ${ }^{3}$ CIEMAT". ${ }^{4}$ Comisión Nacional de Investigación y Desarrollo Aeroespacial". ${ }^{5}$ Departamento de Ciencias de la Atmósfera y los Océanos, Facultad de Ciencias Exactas y Naturales, Universidad de Buenos Aires.". ${ }^{6}$ Departamento de Física (FCEN,UBA)". ${ }^{7}$ Departamento Física Médica, CNEA-CONICET-UNSAM". ${ }^{8}$ Escuela Politécnica Nacional". ${ }^{9}$ Escuela Superior Politécnica de Chimborazo". ${ }^{10}$ European Soutern Observatory (ESO)". ${ }^{11}$ Facultad de Ciencias Exactas y Tecnología (FACET) - Universidad Nacional de Tucumán (UNT)". ${ }^{12}$ Instituto Antártico Argentino, Dirección Nacional del Antartico, Instituto de Astronomía y Física del Espacio (UBA-CONICET)". ${ }^{13}$ Instituto de Astronomía y Física del Espacio, IAFE (UBA-CONICET)". ${ }^{14}$ Instituto de Tecnologías en Detección y Astropartículas (CNEA, CONICET,UNSAM)". ${ }^{16}$ Universidad Autónoma de Chiapas". ${ }^{17}$ Universidad de La Serena". ${ }^{18}$ Universidad de Pamplona". ${ }^{19}$ Universidad de San Carlos". ${ }^{20}$ Universidad de Valparaíso". ${ }^{21}$ Universidad de Viña del Mar". ${ }^{22}$ Universidad del Valle de Guatemala". ${ }^{23}$ Universidad Industrial de Santander". ${ }^{24}$ Universidad Mayor de San Andrés". ${ }^{25}$ Universidad Nacional de Ingeniería". ${ }^{26}$ Universidad San Francisco de Quito". ${ }^{27}$ Universidade Estadual de Campinas". ${ }^{28}$ Universidade Federal de Campina Grande". ${ }^{29}$ Universidade Federal do ABC". ${ }^{30}$ Universidade Federal do Recôncavo da Bahia". ${ }^{31}$ Université Libre de Bruxelles, Brussels, Belgium.". 